\title{
Design, Synthesis, Biological Evaluation, and Molecular Docking Studies of Quinolone Derivatives as Potential Antitumor Topoisomerase I Inhibitors
}

\author{
Kai-jun Shou, ${ }^{a}$ Jie Li, ${ }^{b}$ Yi Jin, ${ }^{b}$ and Yan-wen $\mathrm{Lv}^{*, b, c}$ \\ ${ }^{a}$ People's Hospital of Zhuji; Zhuji 312000, China: ${ }^{b}$ College of Chemistry and Materials Engineering, Quzhou \\ University; Quzhou 324000, China: and ${ }^{c}$ Key Laboratory of Pharmaceutical Engineering of Ministry of Education, \\ College of Pharmaceutical Sciences, Zhejiang University of Technology; Hangzhou 310014, China. \\ Received January 11, 2013; accepted April 1, 2013; advance publication released online April 5, 2013
}

\begin{abstract}
A novel series of quinolone derivatives (6a-n) were designed and synthesized, and their biological activities were evaluated as potential antitumor topoisomerase I (Top I) inhibitors. Among these compounds, 6j exhibited the most potent antitumor activities against multiple cancer cell lines. Docking simulation was performed to insert compound $6 \mathrm{j}$ into the crystal structure of DNA-Top I to determine the probable binding model.
\end{abstract}

Key words quinolone derivative; antitumor; topoisomerase I; molecular docking

DNA-topoisomerase I (Top I) is an important enzyme to relax supercoiled DNA for transcription, replication, and mitosis. ${ }^{1)}$ Top I relaxes DNA by producing reversible single-strand DNA breaks. The generally accepted mechanism of Top I action involves a nucleophilic attack by the catalytic tyrosine 723 residue of Top I on the phosphate group at 3 '-end of the broken DNA strand, which leads to the break of the DNA phosphodiester bond and the formation of a binary Top I-DNA covalent complex (Top I-DNAcc). The rotation of the $5^{\prime}$-end around the intact strand allows for relaxation of the supercoil. $^{1,2)}$ As it plays a pivotal role in cellular proliferation, Top I is often overexpressed in human tumors. Thus, DNA-Top I has been identified as a promising cancer therapeutic target. ${ }^{3)}$ Camptothecin (CPT) (1) ${ }^{4)}$ and its clinically used analogues, topotecan (2) and irinotecan $(3)^{5,6)}$ (Fig. 1), were found to inhibit Top I activity by intercalating into the cleavage complex and preventing the relegation step of the catalytic cycle. As a result, the covalent Top I-DNA adduct produces collisions with advancing replication forks and transcription complexes, which triggers irreversible DNA damage and apoptosis. ${ }^{7,8}$

Although the camptothecins possess potent antitumor activity, they also suffer from well-identified drawbacks, including solubility and bioactivity, dose-limiting toxicity. ${ }^{7-10)}$ Additionally, the E-ring lactone of camptothecin is readily opened to its hydroxycarboxylate form in vivo. ${ }^{11)}$ This form is less active and binds strongly to human blood proteins. ${ }^{12)}$ Thus, these problems recommended further development of other nonCPT Top I inhibitors with better pharmacokinetic features.

The non-CPT Top I inhibitors include indenoisoquinolines, ${ }^{13-15)}$ indolocarbazones, ${ }^{16)}$ saintopin, ${ }^{17)}$ benzophenazines, ${ }^{18)}$ terpyridines, ${ }^{19)}$ and 3 -arylisoquinolines. ${ }^{20)}$ The successful clinical cases of the non-CPT derivatives were attributed to the better chemical stability at longer lifetimes of the trapped cleavage complex of the non-CPT Top I inhibitors at the absence of a lactone ring in their skeleton. ${ }^{21)}$

Quinolones are among the most common frameworks present in bioactive molecules and hence represent an attractive starting point for the design of combinatorial libraries. ${ }^{22)} \mathrm{Re}$ cently, You and co-workers ${ }^{23}$ discovered series of quinolone

The authors declare no conflict of interest.

*To whom correspondence should be addressed. e-mail: lyw@qzu.zj.cn derivatives as potential Top I inhibitors by scaffold modification. In addition, Al-Trawneh et al. ${ }^{24)}$ also reported that tetracyclic fluoroquinolones exhibited high antiproliferative activity against breast MCF-7 and lung A549 tumor cell lines.

We attempted to design quinolone derivatives by scaffold modification (Fig. 2). Fragment A was designed based on Top I inhibitors CPUY013 ${ }^{25)}$ and A-62176. ${ }^{26)}$ Fragment B was designed on the basis of Top I-DNA and CPT binding mode ${ }^{1)}$ which showed that the amino acid residues $\mathrm{Asn}^{722}$ provides interactions with the A-ring of CPT. The target scaffold was a combination of fragment $\mathrm{A}$ and $\mathrm{B}$, with water soluble sulfonyl guanidine as a linker. Herein, we report the synthesis and in vitro evaluation of the antitumor properties of novel $N$-substituted piperazinylquinolone derivatives $(\mathbf{6 a}-\mathbf{n})$. The probable mechanism of the active compound $(\mathbf{6 j})$ was also examined by docking studies.

\section{Results and Discussion}

Chemistry $N$-Substituted piperazinylquinolone derivatives $(\mathbf{6 a}-\mathbf{n})$ were prepared via a two-step one-pot tandem process. The general synthetic pathway of target compounds is outlined in Chart 1. The description of substituents and yields are listed in Table 1.

The reaction of arylsulfonyl chloride (4) with cyanamide

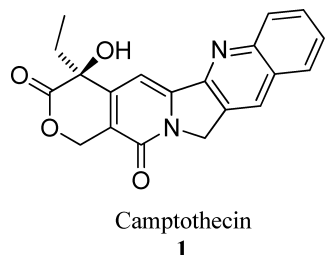

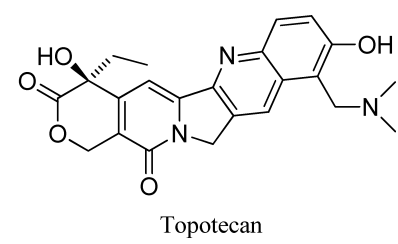

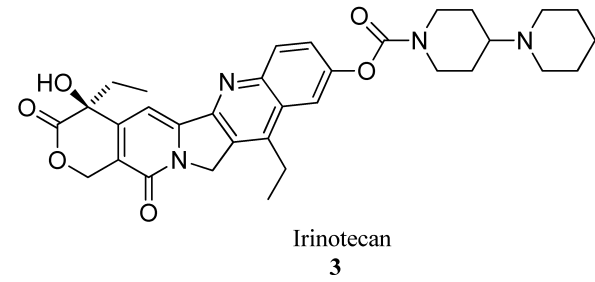

Fig. 1. Structures of $\mathbf{1}$ and Its Derivatives 


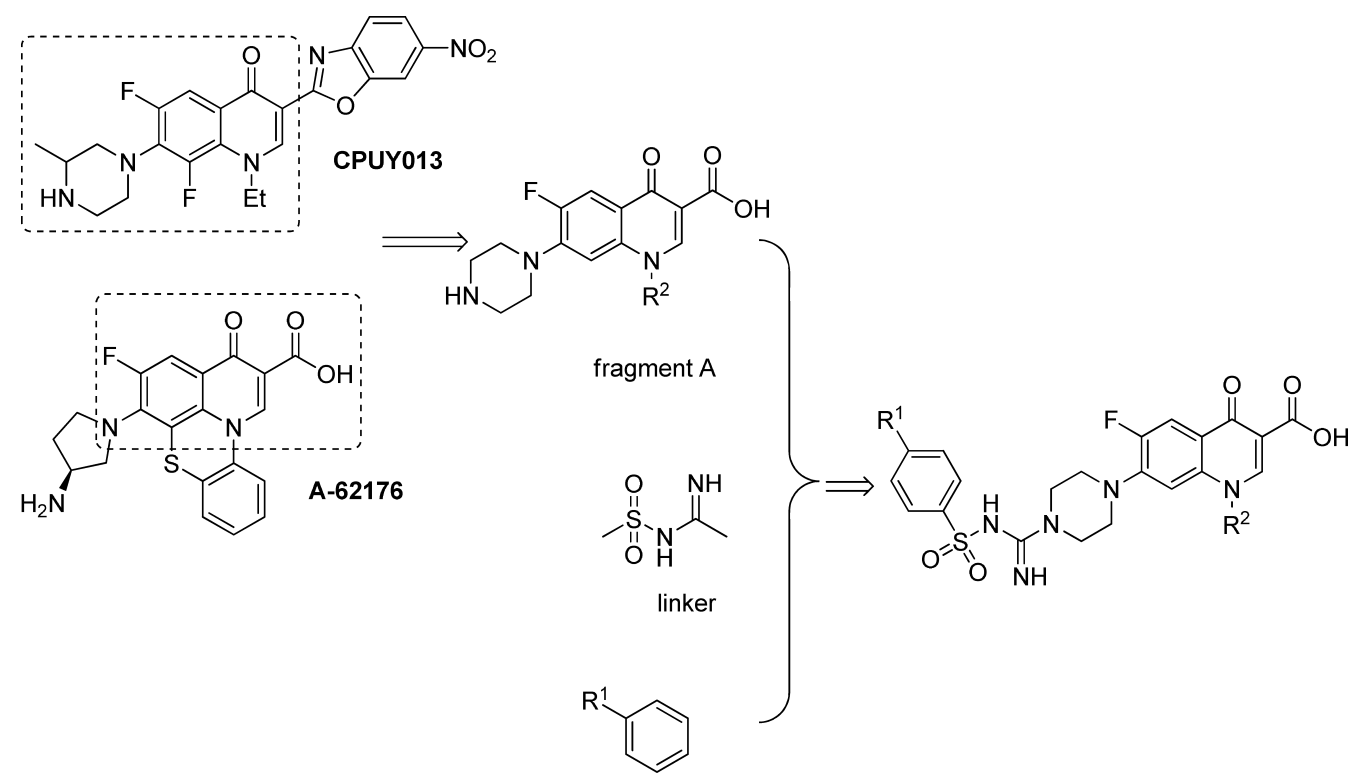

fragment B

Fig. 2. Compound Generation by Scaffold Modification<smiles>[R]c1cccc(S(=O)(=O)NC(=N)N2CCN(c3cc4c(cc3F)c(=O)c(C(=O)O)cn4[R2])CC2)c1</smiles>

Reagents and conditions: (a) $\mathrm{NH}_{2} \mathrm{CN}\left(50 \%\right.$ ), butanone, $40-60^{\circ} \mathrm{C}, 3 \mathrm{~h}$; (b) norfloxacin or ciprofloxacin, $80^{\circ} \mathrm{C}, 3 \mathrm{~h}$.

Chart 1. General Synthesis of Compounds $(\mathbf{6 a}-\mathbf{n})$

to form intermediates $\mathbf{5}$ is a typical nucleophilic substitution reaction. The relatively weak nucleophilicity of the cyanamide and the presence of dicyanamide may lead to byproducts. The product arylsulfonyl cyanamide (5) can also react with cyanamide to form byproducts. Therefore the reaction conditions have to be carefully controlled. The target compound $\mathbf{6}$ was prepared by a subsequent nucleophilic addition of commercially available norfloxacin hydrochloride or ciprofloxacin hydrochloride with $\mathbf{5}$ in one-pot with yields ranging from $42 \%$ to $85 \%$ (Table 1). The intermediates $\mathbf{5}$ are soluble in aqueous medium, while the target compounds are not very soluble in the reaction medium. Thus the $\mathrm{pH}$ of each reaction mixture has to be well adjusted to precipitate the product.

Biological Activity and Discussion Evaluation of the synthetic $N$-substituted piperazinylquinolone derivatives (6an) for antiproliferative activity was performed by 3-(4,5-dimethylthiazol-2-yl)-2,5-diphenyltetrazolium bromide (MTT) assay using three different human cancer cell lines, human lung carcinoma cell (A549), human promyelocytic leukemia cell (HL-60) and human cervical cancer cell (Hela). The assessments of antiproliferative activities were expressed as the concentration inhibiting $50 \%$ of cancer cell growth $\left(\mathrm{IC}_{50}\right)$. The Top I inhibitory activity assays was carried out using a topoisomerase I drug screening kit. The results were summarized in Table 2.

As shown in Table 2, these $N$-substituted piperazinylquinolone derivatives bearing the fluoro atom and piperazine on the
Table 1. Structure and Synthetic Yield of the Target Compounds

\begin{tabular}{clll}
\hline \hline Compound & \multicolumn{1}{c}{$\mathrm{R}^{1}$} & \multicolumn{1}{c}{$\mathrm{R}^{2}$} & Yield (\%) \\
\hline $\mathbf{6 a}$ & $\mathrm{H}$ & $\mathrm{Et}$ & 55 \\
$\mathbf{6 b}$ & $\mathrm{H}$ & $c-\mathrm{C}_{3} \mathrm{H}_{5}$ & 70 \\
$\mathbf{6 c}$ & $4-\mathrm{Me}$ & $\mathrm{Et}$ & 76 \\
$\mathbf{6 d}$ & $4-\mathrm{Me}$ & $c-\mathrm{C}_{3} \mathrm{H}_{5}$ & 85 \\
$\mathbf{6 e}$ & $4-\mathrm{Cl}$ & $\mathrm{Et}$ & 75 \\
$\mathbf{6 f}$ & $4-\mathrm{Cl}$ & $c-\mathrm{C}_{3} \mathrm{H}_{5}$ & 84 \\
$\mathbf{6 g}$ & $4-\mathrm{CF}_{3}$ & $\mathrm{Et}$ & 76 \\
$\mathbf{6 h}$ & $4-\mathrm{CF}_{3}$ & $c-\mathrm{C}_{3} \mathrm{H}_{5}$ & 83 \\
$\mathbf{6 i}$ & $2-\mathrm{Cl}$ & $\mathrm{Et}$ & 74 \\
$\mathbf{6 j}$ & $2-\mathrm{Cl}$ & $c-\mathrm{C}_{3} \mathrm{H}_{5}$ & 58 \\
$\mathbf{6 k}$ & $2-\mathrm{CF}_{3}$ & $\mathrm{Et}$ & 71 \\
$\mathbf{6 l}$ & $2-\mathrm{CF}_{3}$ & $c-\mathrm{C}_{3} \mathrm{H}_{5}$ & 76 \\
$\mathbf{6 m}$ & $3-\mathrm{Cl}^{\mathbf{6}}$ & $\mathrm{Et}$ & 58 \\
$\mathbf{6 n}$ & $3-\mathrm{Cl}$ & $c-\mathrm{C}_{3} \mathrm{H}_{5}$ & 42 \\
\hline
\end{tabular}

skeleton exhibited remarkable antiproliferative effects. Among them, compound $\mathbf{6 j}$ displayed the most potent inhibitory activity $\left(\mathrm{IC}_{50}=0.072 \mu \mathrm{M}\right.$ for $\mathrm{A} 549, \mathrm{IC}_{50}=0.043 \mu \mathrm{M}$ for HL-60 and $\mathrm{IC}_{50}=0.039 \mu \mathrm{M}$ for Hela), similar to the positive control Irinotecan $\left(\mathrm{IC}_{50}=0.032 \mu \mathrm{M}\right.$ for A549, $\mathrm{IC}_{50}=0.044 \mu \mathrm{M}$ for HL-60 and $\mathrm{IC}_{50}=0.038 \mu \mathrm{M}$ for Hela, respectively). As expected, compound 6j showed excellent Top I inhibitory activity $(++++)$ comparable to Irinotecan. Consistency between cytotoxicities and Top I inhibitory activities was observed in these series, sug- 
Table 2. $\mathrm{IC}_{50}$ Cytotoxicity and Top I Inhibitory of Target Compounds

\begin{tabular}{|c|c|c|c|c|}
\hline \multirow{2}{*}{ Compd. } & \multicolumn{3}{|c|}{$\mathrm{IC}_{50}(\mu \mathrm{M})$} & \multirow{2}{*}{ Top $\mathrm{I}^{a}$} \\
\hline & A549 & HL-60 & Hela & \\
\hline $6 a$ & 0.161 & 0.104 & 0.093 & + \\
\hline $6 b$ & 0.153 & 0.099 & 0.087 & + \\
\hline $6 c$ & $>0.193$ & 0.146 & 0.122 & - \\
\hline 6d & $>0.189$ & 0.139 & 0.118 & - \\
\hline $6 e$ & 0.130 & 0.084 & 0.063 & ++ \\
\hline $6 f$ & 0.123 & 0.079 & 0.068 & ++ \\
\hline $6 \mathrm{~g}$ & 0.101 & 0.065 & 0.057 & ++ \\
\hline $6 \mathrm{~h}$ & 0.096 & 0.062 & 0.055 & ++ \\
\hline $6 i$ & 0.079 & 0.052 & 0.045 & ++++ \\
\hline $6 \mathbf{j}$ & 0.071 & 0.043 & 0.032 & ++++ \\
\hline $6 \mathrm{k}$ & 0.101 & 0.066 & 0.058 & +++ \\
\hline 61 & 0.098 & 0.063 & 0.056 & +++ \\
\hline $6 \mathrm{~m}$ & 0.089 & 0.059 & 0.052 & +++ \\
\hline $6 n$ & 0.085 & 0.056 & 0.048 & +++ \\
\hline Irinotecan & 0.032 & 0.044 & 0.038 & ++++ \\
\hline Norfloxacin & $>0.313$ & $>0.313$ & $>0.313$ & - \\
\hline Ciprofloxacin & $>0.302$ & $>0.302$ & $>0.302$ & - \\
\hline
\end{tabular}

a) Activity is expressed semi-quantitatively as follows: - no activity, + very weak activity, ++ weak activity, +++ lower activity than Irinotecan, ++++ similar or greater activity than Irinotecan.

gesting that the target of $N$-substituted piperazinylquinolones is limited to Top I.

Subsequently, structure-activity relationships (SAR) studies were inferred from Table 2. In general, target compounds with $\mathrm{R}^{2}=$ cyclopropyl shown more potent activities than those with $\mathrm{R}^{2}=$ ethyl at $N-1$ position. And the substituent group on the benzene ring had decisive affect on the $\mathrm{IC}_{50}$ value. Compounds with electron-donating group on the benzene ring, such as $\mathbf{6 c}$ and $\mathbf{d}$, shown weaker cytotoxic activities, and electron-withdrawing group such as chlorine or trifluoromethyl on the benzene ring would increase the activities. Moreover, the position of substituent $\left(\mathrm{R}^{1}\right)$ on the benzene ring also influenced the activities. Compounds with ortho substituent (6i-l) shown more potent activities than those with meta $(\mathbf{6 m}, \mathbf{n})$ or para $(\mathbf{1} \mathbf{e}-\mathbf{h})$ substituent.

Docking Study Molecular docking is an application wherein molecular modeling techniques are used to predict how a protein interacts with small molecules. ${ }^{27}$ In the present study, to gain better understanding on the interactions between target compound and Top I (PDB code: 1T8I) and to explore their binding mode, a docking study was performed using the CDOCKER protocol in Discovery Studio 2.1 (Discovery Studio 2.1, Accelrys, Inc., San Diego, CA, U.S.A.).

Docking model illustrates that the quinolone derivatives of $\mathbf{6 j}$ was well positioned in the binding sites of DNA-Top I complex (Fig. 3). The structural model suggested that one of the two sulfonyl oxygens, fluoro atom on the quinolone skeleton and quinolone ring, interacted with enzyme (Fig. 3a). One of the sulfonyl oxygen formed one hydrogen bond with the amino hydrogen of Lys425. And the fluoro atom on the quinolone ring of compound $\mathbf{6 j}$ associates with Arg364 by two hydrogen bonds. The quinolone skeleton of compound $\mathbf{6 j}$ is calculated to interact with Arg364 and is likely stabilized by $\pi$-stacking interactions.

All the amino acid residues which had interactions with compound $\mathbf{6 j}$ were displayed in Fig. 3b. The enzyme surface model was shown in Fig. $3 \mathrm{c}$, which revealed that the molecule was well embedded in the active pocket. This molecular dock- ing result, along with the biological assays, suggested that $\mathrm{N}$-substituted piperazinylquinolone derivatives were potential Top I inhibitors as anti-cancer agents.

\section{Conclusion}

In our present work, a novel series of quinolone derivatives $(\mathbf{6} \mathbf{a}-\mathbf{n})$ have been synthesized and evaluated. These compounds exhibited potent antiproliferative activities against A549, HL-60, and Hela cells. Among them, compound $\mathbf{6 j}$ demonstrated strong cytotoxicity as well as potent Top I inhibitory activity. Consistency between cytotoxicities and Top I inhibitory activities suggesting that the target of $N$-substituted piperazinylquinolones is limited to Top I. Molecular docking studies shown that compound $\mathbf{6 j}$ bound to the DNA-Top I binding site by three hydrogen bonds and two $\pi$-cation interactions which might play crucial roles in its antiproliferative activities. Further discovery of more enhanced $N$-substituted piperazinylquinolone derivatives with better anticancer activity and lower toxicity and side-effects is under the way.

\section{Experimental}

Melting points were determined on Büchi B-540 melting point apparatus and are uncorrected. ${ }^{1} \mathrm{H}-\mathrm{NMR}(300 \mathrm{MHz})$ spectra were recorded on a Bruker AV $300 \mathrm{MHz}$ spectrometer. Mass spectra were obtained on a Thermo Finnigan LCQ-Advantage spectrometer (electrospray ionization (ESI), atmospheric-pressure chemical ionization (APCI)). High resolution (HR)-MS was carried out on an APEX (Bruker) mass III spectrometer. The compounds were dissolved in DMSO- $d_{6}$. Chemical shifts, $\delta$, are given in ppm relative to tetramethylsilane (TMS) $(\delta=0)$ and are referenced by using the residual undeuterated solvent signal. Coupling constants, J, are reported in $\mathrm{Hz}$, multiplicities being marked as: singlet (s), broad singlet (brs), doublet (d), triplet (t), quartet (q), multiplet (m).

General Experimental Procedure Benzenesulfonyl chloride $(0.01 \mathrm{~mol})$ in butanone $(30 \mathrm{~mL})$ was heated with stirring to $40^{\circ} \mathrm{C}$, and cyanamide solution $(50 \%)$ was added dropwise during $30 \mathrm{~min}$. The temperature was raised to $60^{\circ} \mathrm{C}$ and stir- 


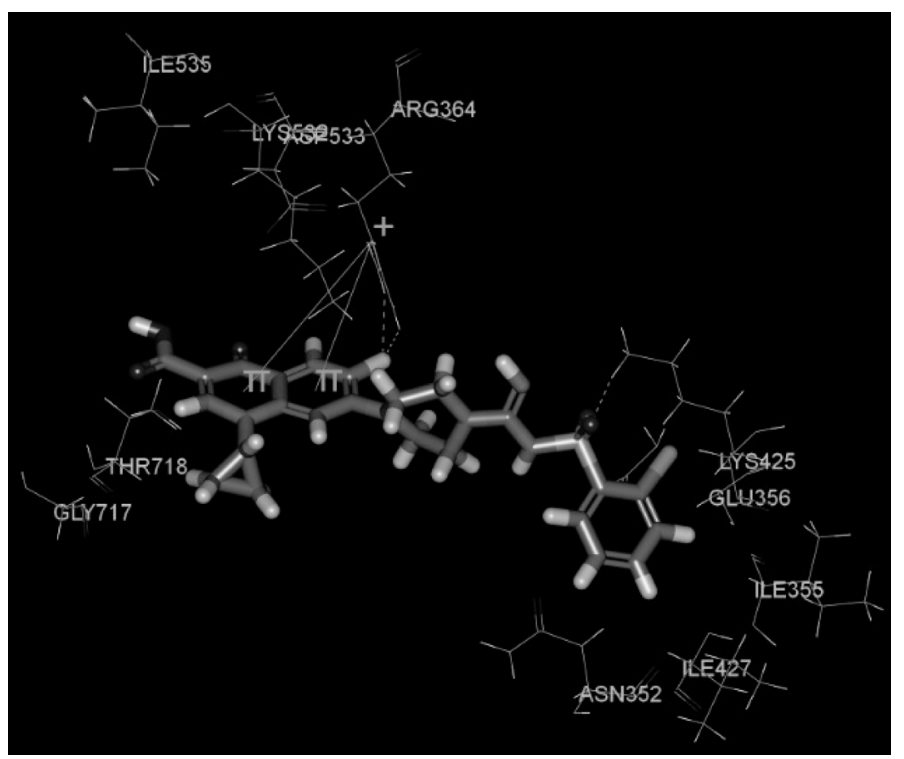

Fig. 3a. Compound $\mathbf{6 j}$ Bound into Top I

The dotted lines show the hydrogen bonds and the solid lines show the $\pi$-cation interactions.

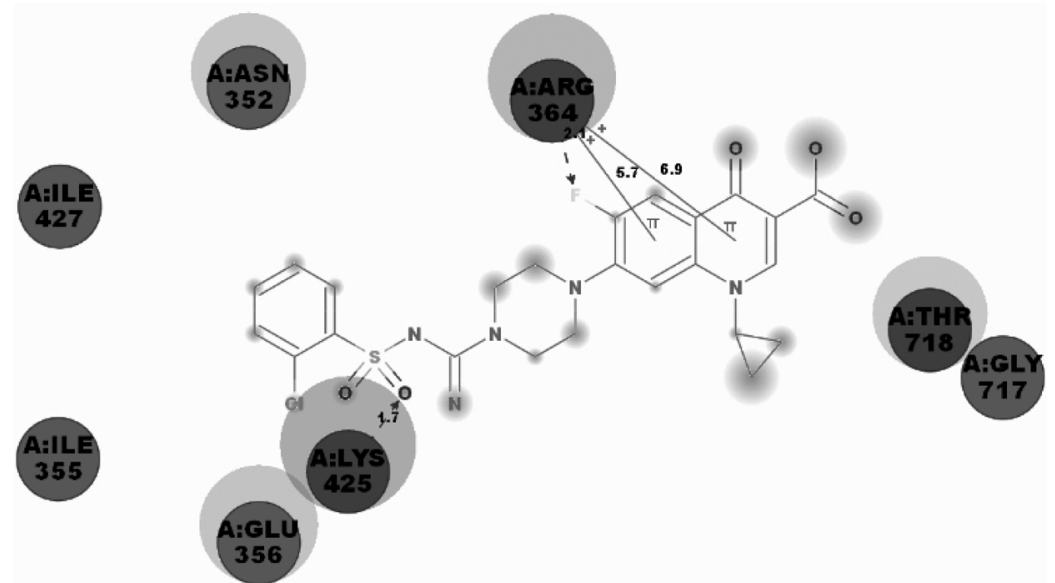

Fig. 3b. 2D Ligand Interaction Diagram of Compound 6j with Top I Using Discovery Studio Program with the Essential Amino Acid Residues at the Binding Site Are Tagged in Circles

ring continued for $3 \mathrm{~h}$. After cooling the reaction mixture to below $30^{\circ} \mathrm{C}$, norfloxacin hydrochloride or ciprofloxacin hydrochloride monohydrate $(0.008 \mathrm{~mol})$ was added. The mixture was heated to $80^{\circ} \mathrm{C}$ and maintained for $3 \mathrm{~h}$. After cooling to below $40^{\circ} \mathrm{C}$, the reaction mixture was poured into cold water while stirring, white crystals or powders were precipitated, filtered, washed with water, and dried at $80^{\circ} \mathrm{C}$. Analytically pure samples were obtained by recrystallization from aqueous ethanol.

1-Ethyl-6-fluoro-4-oxo-7-(4-(N-(phenylsulfonyl)carbamimidoyl)piperazin-1-yl)-1,4-dihydroquinoline-3carboxylic Acid (6a): White crystals, mp $221-225^{\circ} \mathrm{C} ; 55 \%$ yield; ${ }^{1} \mathrm{H}-\mathrm{NMR}$ (DMSO- $\left.d_{6}, 300 \mathrm{MHz}\right) \delta(\mathrm{ppm}): 1.41$ (t, $3 \mathrm{H}$, $J=4.5 \mathrm{~Hz}$ ), 3.19 (m, 4H), 3.43 (m, 4H), 4.58 (q, 2H, $J=4.5 \mathrm{~Hz}$ ), $7.17(\mathrm{~d}, 1 \mathrm{H}, J=3.0 \mathrm{~Hz}), 7.35(\mathrm{~m}, 2 \mathrm{H}), 7.58(\mathrm{~m}, 3 \mathrm{H}), 7.86(\mathrm{~d}$, $1 \mathrm{H}, J=9.0 \mathrm{~Hz}$ ), 8.82 (s, 1H), 8.91 (brs, 2H), 15.05 (brs, 1H); HR-MS Calcd for $\mathrm{C}_{23} \mathrm{H}_{25} \mathrm{FN}_{5} \mathrm{O}_{5} \mathrm{~S}\left[\mathrm{M}+\mathrm{H}^{+}\right]$: 502.1560. Found: 502.1554 .

1-Cyclopropyl-6-fluoro-4-oxo-7-(4-( $N$-(phenylsulfonyl)carbamimidoyl)piperazin-1-yl)-1,4-dihydroquinoline-3- carboxylic Acid (6b): White crystals, mp 295-298 ${ }^{\circ} \mathrm{C} ; 70 \%$ yield; ${ }^{1} \mathrm{H}-\mathrm{NMR}$ (DMSO- $\left.d_{6}, 300 \mathrm{MHz}\right) \delta(\mathrm{ppm}): 1.18(\mathrm{~m}, 2 \mathrm{H})$, $1.33(\mathrm{~m}, 2 \mathrm{H}), 3.33(\mathrm{~m}, 4 \mathrm{H}), 3.53(\mathrm{~m}, 4 \mathrm{H}), 3.83(\mathrm{~m}, 1 \mathrm{H}), 7.28$ (d, $1 \mathrm{H}, J=3.0 \mathrm{~Hz}), 7.33-7.42(\mathrm{~m}, 2 \mathrm{H}), 7.48-7.65(\mathrm{~m}, 3 \mathrm{H}), 7.90$ (d, $1 \mathrm{H}, J=9.0 \mathrm{~Hz}), 8.63$ (s, 1H), 9.23 (brs, 2H), 15.05 (brs, $1 \mathrm{H})$; HR-MS Calcd for $\mathrm{C}_{24} \mathrm{H}_{25} \mathrm{FN}_{5} \mathrm{O}_{5} \mathrm{~S}\left[\mathrm{M}+\mathrm{H}^{+}\right]$: 514.1560 . Found: 514.1553.

1-Ethyl-6-fluoro-4-oxo-7-(4-(N-tosylcarbamimidoyl)piperazin-1-yl)-1,4-dihydroquinoline-3-carboxylic Acid (6c): White crystals, mp $257-260^{\circ} \mathrm{C} ; 76 \%$ yield; ${ }^{1} \mathrm{H}-\mathrm{NMR}$ (DMSO$\left.d_{6}, 300 \mathrm{MHz}\right) \delta(\mathrm{ppm}): 1.41$ (t, 3H, $\left.J=4.5 \mathrm{~Hz}\right), 2.45$ (s, 3H), 3.19 (m, 4H), 3.43 (m, 4H), 4.58 (q, 2H, J=4.5 Hz), 7.17 (d, 1H, J= $3.0 \mathrm{~Hz}), 7.35(\mathrm{~m}, 2 \mathrm{H}), 7.58(\mathrm{~m}, 3 \mathrm{H}), 7.86(\mathrm{~d}, 1 \mathrm{H}, J=9.0 \mathrm{~Hz})$, 8.82 (s, 1H), 8.91 (brs, 2H), 15.05 (brs, 1H); HR-MS Calcd for $\mathrm{C}_{24} \mathrm{H}_{27} \mathrm{FN}_{5} \mathrm{O}_{5} \mathrm{~S}\left[\mathrm{M}+\mathrm{H}^{+}\right]$: 516.1717. Found: 516.1724.

1-Cyclopropyl-6-fluoro-4-oxo-7-(4-( $N$-tosylcarbamimidoyl)piperazin-1-yl)-1,4-dihydroquinoline-3-carboxylic Acid (6d): White crystals, mp $262-265^{\circ} \mathrm{C} ; 85 \%$ yield; ${ }^{1} \mathrm{H}-\mathrm{NMR}$ (DMSO$\left.d_{6}, 300 \mathrm{MHz}\right) \delta$ (ppm): $1.18(\mathrm{~m}, 2 \mathrm{H}), 1.33$ (m, 2H), 2.45 (s, $3 \mathrm{H}), 3.33(\mathrm{~m}, 4 \mathrm{H}), 3.53(\mathrm{~m}, 4 \mathrm{H}), 3.83(\mathrm{~m}, 1 \mathrm{H}), 7.22(\mathrm{~d}, 1 \mathrm{H}$, 


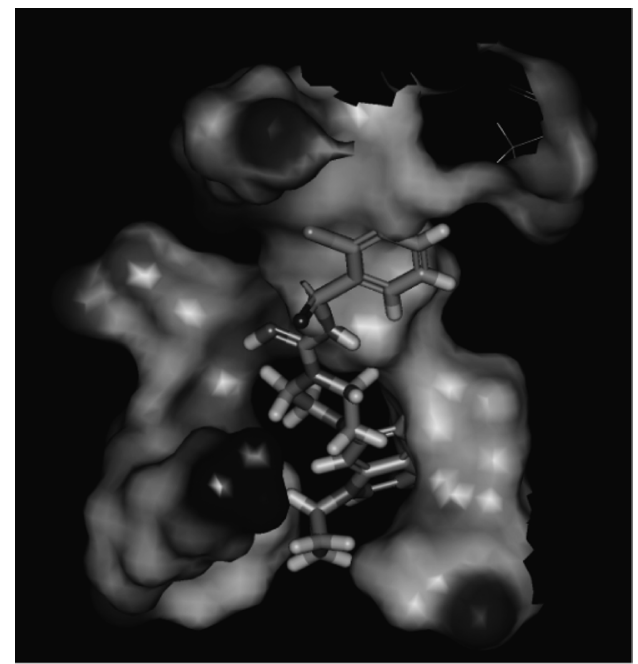

Fig. 3c. 3D Model of the Interaction between Compound $\mathbf{6 j}$ and Top I Bonding Site

The protein is represented by molecular surface. Compound $\mathbf{6} \mathbf{j}$ is depicted by balls.

$J=3.0 \mathrm{~Hz}), 7.38(\mathrm{~m}, 2 \mathrm{H}), 7.58(\mathrm{~m}, 2 \mathrm{H}), 7.86(\mathrm{~d}, 1 \mathrm{H}, J=9.0 \mathrm{~Hz})$, 8.63 (s, 1H), 9.23 (brs, 2H), 15.05 (brs, 1H); HR-MS Calcd for $\mathrm{C}_{25} \mathrm{H}_{27} \mathrm{FN}_{5} \mathrm{O}_{5} \mathrm{~S}\left[\mathrm{M}+\mathrm{H}^{+}\right]$: 528.1717. Found: 528.1723 .

7-(4-(N-(4-Chlorophenylsulfonyl)carbamimidoyl)piperazin-1-yl)-1-ethyl-6-fluoro-4-oxo-1,4-dihydroquinoline-3carboxylic Acid (6e): Light yellow crystals, mp 297-299 ${ }^{\circ}$; $75 \%$ yield; ${ }^{1} \mathrm{H}-\mathrm{NMR}$ (DMSO- $\left.d_{6}, 300 \mathrm{MHz}\right) \delta$ (ppm): 1.41 (t, 3H, J=4.5 Hz), $3.19(\mathrm{~m}, 4 \mathrm{H}), 3.43(\mathrm{~m}, 4 \mathrm{H}), 4.58(\mathrm{q}, 2 \mathrm{H}$, $J=4.5 \mathrm{~Hz}), 7.17(\mathrm{~d}, 1 \mathrm{H}, J=3.0 \mathrm{~Hz}), 7.46(\mathrm{~m}, 2 \mathrm{H}), 7.66(\mathrm{~m}, 2 \mathrm{H})$, 7.90 (d, 1H, J=9.0 Hz), 8.95 (s, 1H), 9.11 (br s, 2H), 15.10 (brs, $1 \mathrm{H}$ ); HR-MS Calcd for $\mathrm{C}_{23} \mathrm{H}_{24} \mathrm{ClFN}_{5} \mathrm{O}_{5} \mathrm{~S}\left[\mathrm{M}+\mathrm{H}^{+}\right]$: 536.1171 . Found: 536.1164 .

7-(4-(N-(4-Chlorophenylsulfonyl)carbamimidoyl)piperazin-1-y1)-1-cyclopropyl-6-fluoro-4-oxo-1,4dihydroquinoline-3-carboxylic Acid (6f): Light yellow crystals, mp $288-291{ }^{\circ} \mathrm{C} ; 84 \%$ yield; ${ }^{1} \mathrm{H}-\mathrm{NMR}$ (DMSO- $d_{6}$, $300 \mathrm{MHz}) \delta(\mathrm{ppm}): 1.18(\mathrm{~m}, 2 \mathrm{H}), 1.33(\mathrm{~m}, 2 \mathrm{H}), 3.33(\mathrm{~m}, 4 \mathrm{H})$, $3.53(\mathrm{~m}, 4 \mathrm{H}), 3.83(\mathrm{~m}, 1 \mathrm{H}), 7.28(\mathrm{~d}, 1 \mathrm{H}, J=3.0 \mathrm{~Hz}), 7.36(\mathrm{~m}$, 2H), $7.60(\mathrm{~m}, 2 \mathrm{H}), 7.90(\mathrm{~d}, 1 \mathrm{H}, J=9.0 \mathrm{~Hz}), 8.63(\mathrm{~s}, 1 \mathrm{H}), 9.23$ (brs, 2H), 15.05 (brs, 1H); HR-MS Calcd for $\mathrm{C}_{24} \mathrm{H}_{24} \mathrm{ClFN}_{5} \mathrm{O}_{5} \mathrm{~S}$ $\left[\mathrm{M}+\mathrm{H}^{+}\right.$]: 548.1171. Found: 548.1179.

1-Ethyl-6-fluoro-4-oxo-7-(4-( $N$-(4-(trifluoromethyl)phenylsulfonyl)carbamimidoyl)piperazin-1-yl)-1,4dihydroquinoline-3-carboxylic Acid (6g): White-like crystals, mp 217-220 ${ }^{\circ} \mathrm{C}$; 76\% yield; ${ }^{1} \mathrm{H}-\mathrm{NMR}$ (DMSO- $\left.d_{6}, 300 \mathrm{MHz}\right) \delta$ (ppm): $1.41(\mathrm{t}, 3 \mathrm{H}, J=4.5 \mathrm{~Hz}), 3.19\left(\mathrm{~m}, 4 \mathrm{H},-\mathrm{CH}_{2}\right), 3.43(\mathrm{~m}$, $4 \mathrm{H}), 4.58(\mathrm{q}, 2 \mathrm{H}, J=4.5 \mathrm{~Hz}), 7.17(\mathrm{~d}, 1 \mathrm{H}, J=3.0 \mathrm{~Hz}), 7.56(\mathrm{~m}$, 2H), $7.90(\mathrm{~d}, 1 \mathrm{H}, J=9.0 \mathrm{~Hz}), 8.22(\mathrm{~m}, 2 \mathrm{H}), 8.86(\mathrm{~s}, 1 \mathrm{H}), 9.15$ (brs, 2H), 15.08 (brs, 1H); HR-MS Calcd for $\mathrm{C}_{24} \mathrm{H}_{24} \mathrm{~F}_{4} \mathrm{~N}_{5} \mathrm{O}_{5} \mathrm{~S}$ $\left[\mathrm{M}+\mathrm{H}^{+}\right]:$570.1434. Found: 570.1427.

1-Cyclopropyl-6-fluoro-4-oxo-7-(4-(N-(4-(trifluoromethyl)phenylsulfonyl)carbamimidoyl)piperazin-1-yl)-1,4dihydroquinoline-3-carboxylic Acid (6h): White-like crystals, mp 248-251 ${ }^{\circ} \mathrm{C}$; 83\% yield; ${ }^{1} \mathrm{H}-\mathrm{NMR}$ (DMSO- $d_{6}, 300 \mathrm{MHz}$ ) $\delta$ (ppm): $1.18(\mathrm{~m}, 2 \mathrm{H}), 1.33(\mathrm{~m}, 2 \mathrm{H}), 3.33(\mathrm{~m}, 4 \mathrm{H}), 3.53(\mathrm{~m}$, $4 \mathrm{H}), 3.83(\mathrm{~m}, 1 \mathrm{H}), 7.28(\mathrm{~d}, 1 \mathrm{H}, J=3.0 \mathrm{~Hz}), 7.47(\mathrm{~m}, 2 \mathrm{H}), 7.90$ (d, $1 \mathrm{H}, J=9.0 \mathrm{~Hz}), 8.06(\mathrm{~m}, 2 \mathrm{H}), 8.63(\mathrm{~s}, 1 \mathrm{H}), 9.23$ (brs, 2H), 15.05 (brs, $1 \mathrm{H}$ ); HR-MS Calcd for $\mathrm{C}_{25} \mathrm{H}_{24} \mathrm{~F}_{4} \mathrm{~N}_{5} \mathrm{O}_{5} \mathrm{~S}\left[\mathrm{M}+\mathrm{H}^{+}\right]$: 582.1434. Found: 582.1440.
7-(4-(N-(2-Chlorophenylsulfonyl) carbamimidoyl)piperazin-1-yl)-1-ethyl-6-fluoro-4-oxo-1,4-dihydroquinoline-3carboxylic Acid (6i): Pale yellow crystals, mp $255-258^{\circ} \mathrm{C} ; 74 \%$ yield; ${ }^{1} \mathrm{H}-\mathrm{NMR}$ (DMSO- $\left.d_{6}, 300 \mathrm{MHz}\right) \delta(\mathrm{ppm}): 1.41$ (t, $3 \mathrm{H}$, $J=4.5 \mathrm{~Hz}$ ), 3.19 (m, 4H), 3.43 (m, 4H), 4.58 (q, 2H, $J=4.5 \mathrm{~Hz}$ ), $7.17(\mathrm{~d}, 1 \mathrm{H}, J=3.0 \mathrm{~Hz}), 7.46(\mathrm{~m}, 3 \mathrm{H}), 7.90(\mathrm{~d}, 1 \mathrm{H}, J=9.0 \mathrm{~Hz})$, $8.46(\mathrm{~m}, 1 \mathrm{H}), 8.95$ (s, 1H), 9.11 (brs, 2H), 15.10 (brs, 1H); HR-MS Calcd for $\mathrm{C}_{23} \mathrm{H}_{24} \mathrm{ClFN}_{5} \mathrm{O}_{5} \mathrm{~S}\left[\mathrm{M}+\mathrm{H}^{+}\right]$: 536.1171. Found: 536.1166 .

7-(4-(N-(2-Chlorophenylsulfonyl)carbamimidoyl)piperazin-1-yl)-1-cyclopropyl-6-fluoro-4-oxo-1,4dihydroquinoline-3-carboxylic Acid (6j): Pale yellow crystals, mp $286-289^{\circ} \mathrm{C} ; 58 \%$ yield; ${ }^{1} \mathrm{H}-\mathrm{NMR}$ (DMSO- $d_{6}, 300 \mathrm{MHz}$ ) $\delta$ (ppm): $1.18(\mathrm{~m}, 2 \mathrm{H}), 1.33(\mathrm{~m}, 2 \mathrm{H}), 3.33(\mathrm{~m}, 4 \mathrm{H}), 3.53(\mathrm{~m}$, $4 \mathrm{H}), 3.83(\mathrm{~m}, 1 \mathrm{H}), 7.28(\mathrm{~d}, 1 \mathrm{H}, J=3.0 \mathrm{~Hz}), 7.46(\mathrm{~m}, 3 \mathrm{H}), 7.90$ (d, $1 \mathrm{H}, J=9.0 \mathrm{~Hz}), 8.46(\mathrm{~m}, 1 \mathrm{H}), 8.63(\mathrm{~s}, 1 \mathrm{H}), 9.23$ (br s, 2H), 15.05 (brs, $1 \mathrm{H}$ ); HR-MS Calcd for $\mathrm{C}_{24} \mathrm{H}_{24} \mathrm{ClFN}_{5} \mathrm{O}_{5} \mathrm{~S}\left[\mathrm{M}+\mathrm{H}^{+}\right]$: 548.1171. Found: 548.1169.

1-Ethyl-6-fluoro-4-oxo-7-(4-(N-(2-(trifluoromethyl)phenylsulfonyl)carbamimidoyl)piperazin-1-yl)-1,4dihydroquinoline-3-carboxylic Acid (6k): White-like crystals, mp $218-221^{\circ} \mathrm{C} ; 71 \%$ yield; ${ }^{1} \mathrm{H}-\mathrm{NMR}$ (DMSO- $\left.d_{6}, 300 \mathrm{MHz}\right) \delta$ (ppm): 1.41 (t, 3H, $J=4.5 \mathrm{~Hz}), 3.19(\mathrm{~m}, 4 \mathrm{H}), 3.43(\mathrm{~m}, 4 \mathrm{H}), 4.58$ (q, 2H, $J=4.5 \mathrm{~Hz}), 7.17(\mathrm{~d}, 1 \mathrm{H}, J=3.0 \mathrm{~Hz}), 7.64-7.85(\mathrm{~m}, 3 \mathrm{H})$, $7.90(\mathrm{~d}, 1 \mathrm{H}, J=9.0 \mathrm{~Hz}), 8.50(\mathrm{~m}, 1 \mathrm{H}), 8.95(\mathrm{~s}, 1 \mathrm{H}), 9.11$ (brs, 2H), 15.10 (brs, 1H); HR-MS Calcd for $\mathrm{C}_{24} \mathrm{H}_{24} \mathrm{~F}_{4} \mathrm{~N}_{5} \mathrm{O}_{5} \mathrm{~S}[\mathrm{M}+$ $\mathrm{H}^{+}$]: 570.1434 . Found: 570.1437.

1-Cyclopropyl-6-fluoro-4-oxo-7-(4-( $N$-(2-(trifluoromethyl)phenylsulfonyl)carbamimidoyl)piperazin-1-yl)-1,4dihydroquinoline-3-carboxylic Acid (61): White-like crystals, mp $237-240^{\circ} \mathrm{C}$; $76 \%$ yield; ${ }^{1} \mathrm{H}-\mathrm{NMR}$ (DMSO- $\left.d_{6}, 300 \mathrm{MHz}\right) \delta$ (ppm): $1.18(\mathrm{~m}, 2 \mathrm{H}), 1.33(\mathrm{~m}, 2 \mathrm{H}), 3.33(\mathrm{~m}, 4 \mathrm{H}), 3.53(\mathrm{~m}, 4 \mathrm{H})$, $3.83(\mathrm{~m}, 1 \mathrm{H}), 7.28(\mathrm{~d}, 1 \mathrm{H}, J=3.0 \mathrm{~Hz}), 7.64-7.85(\mathrm{~m}, 3 \mathrm{H}), 7.90$ $(\mathrm{d}, 1 \mathrm{H}, J=9.0 \mathrm{~Hz}), 8.50(\mathrm{~m}, 1 \mathrm{H}), 8.73(\mathrm{~s}, 1 \mathrm{H}), 9.18$ (brs, 2H), 15.05 (brs, 1H); HR-MS Calcd for $\mathrm{C}_{25} \mathrm{H}_{24} \mathrm{~F}_{4} \mathrm{~N}_{5} \mathrm{O}_{5} \mathrm{~S}\left[\mathrm{M}+\mathrm{H}^{+}\right]$: 582.1434. Found: 582.1427.

7-(4-(N-(3-Chlorophenylsulfonyl)carbamimidoyl) piperazin-1-yl)-1-ethyl-6-fluoro-4-oxo-1,4-dihydroquinoline-3carboxylic Acid (6m): White-like crystals, mp $222-225^{\circ} \mathrm{C}$; $58 \%$ yield; ${ }^{1} \mathrm{H}-\mathrm{NMR}$ (DMSO- $\left.d_{6}, 300 \mathrm{MHz}\right) \delta$ (ppm): 1.41 (t, 3H, J=4.5 Hz), $3.19(\mathrm{~m}, 4 \mathrm{H}), 3.43(\mathrm{~m}, 4 \mathrm{H}), 4.58$ (q, 2H, $J=4.5 \mathrm{~Hz}), 7.17(\mathrm{~d}, 1 \mathrm{H}, J=3.0 \mathrm{~Hz}), 7.35-7.60(\mathrm{~m}, 3 \mathrm{H}), 7.86$ (s, 1H), $7.95(\mathrm{~d}, 1 \mathrm{H}, J=9.0 \mathrm{~Hz}), 8.86(\mathrm{~s}, 1 \mathrm{H}), 9.16(\mathrm{brs}, 2 \mathrm{H})$, 15.00 (brs, 1H); HR-MS Calcd for $\mathrm{C}_{23} \mathrm{H}_{24} \mathrm{ClFN}_{5} \mathrm{O}_{5} \mathrm{~S}\left[\mathrm{M}+\mathrm{H}^{+}\right]$: 536.1171. Found: 536.1178 .

7-(4-(N-(3-Chlorophenylsulfonyl)carbamimidoyl)piperazin-1-y1)-1-cyclopropyl-6-fluoro-4-oxo-1,4dihydroquinoline-3-carboxylic Acid (6n): White-like crystals, mp $228-232{ }^{\circ} \mathrm{C} ; 42 \%$ yield; ${ }^{1} \mathrm{H}-\mathrm{NMR}$ (DMSO- $\left.d_{6}, 300 \mathrm{MHz}\right) \delta$ (ppm): 1.18 (m, 2H), 1.33 (m, 2H), $3.33(\mathrm{~m}, 4 \mathrm{H}), 3.53(\mathrm{~m}, 4 \mathrm{H})$, $3.83(\mathrm{~m}, 1 \mathrm{H}), 7.28(\mathrm{~d}, 1 \mathrm{H}, J=3.0 \mathrm{~Hz}), 7.35-7.60(\mathrm{~m}, 3 \mathrm{H}), 7.86$ (s, 1H), $7.90(\mathrm{~d}, 1 \mathrm{H}, J=9.0 \mathrm{~Hz}), 8.63$ (s, 1H), 9.23 (brs, 2H), 15.05 (brs, 1H); HR-MS Calcd for $\mathrm{C}_{24} \mathrm{H}_{24} \mathrm{ClFN}_{5} \mathrm{O}_{5} \mathrm{~S}\left[\mathrm{M}+\mathrm{H}^{+}\right]$: 548.1171. Found: 548.1177.

Anti-proliferation Assay The antiproliferative activities of the prepared compounds against A549, HL-60 and Hala cells were evaluated using a standard MTT-based colorimetric assay. Target tumor cell lines were grown to log phase in RPMI 1640 medium supplemented with $10 \%$ fetal bovine serum. After diluting to $1 \times 10^{6}$ cells $\mathrm{mL}^{-1}$ with the complete medium, $100 \mu \mathrm{L}$ of the obtained cell suspension was added to 
each well of 96-well culture plates. The subsequent incubation was permitted at $37^{\circ} \mathrm{C}, 5 \% \mathrm{CO}_{2}$ atmosphere for $24 \mathrm{~h}$ before the cytotoxicity assessments. Each concentration was in triplicate, and Irinotecan was used as the positive control. After $72 \mathrm{~h}$ incubation at $37^{\circ} \mathrm{C}, 5 \% \mathrm{CO}_{2}$ atmosphere, $10 \mu \mathrm{L}$ of MTT solution in Dulbecco's modified Eagle's medium (DMEM) (Invitrogen, U.S.A.) was added to each well. After three hours incubation at $37^{\circ} \mathrm{C}, 150 \mu \mathrm{L}$ DMSO was added to each well. The plates were then vibrated for $10 \mathrm{~min}$ for complete dissolution. The optical absorbance was measured at $570 \mathrm{~nm}$ on an automated microplate spectrophotometer (Bio-Rad, U.S.A.). In all experiments three replicate wells were used for each drug concentration. The $\mathrm{IC}_{50}$ value was defined as the concentration at which $50 \%$ of the cells could survive. The results were summarized in Table 2.

Docking Simulations The pdb file about the crystal structure of DNA-Top I bound to SA315F (PDB code: 1T8I) ${ }^{28,29)}$ was obtained from the RCSB Protein Data Bank (http://www. pdb.org). The molecular docking procedure was performed by using CDOCKER protocol for receptor-ligand interactions of Discovery Studio 2.5. For ligand preparation, the 3D structures of $\mathbf{6 j}$ were generated and minimized using Discovery Studio 2.5. For protein preparation, the hydrogen atoms were added. The whole DNA-Top I domain defined as a receptor and the site sphere was selected based on the ligand binding location of SA315F, then the SA315F removed and the ligands prepared by us was placed during the molecular docking procedure. CHARMm was selected as the force field. The molecular docking was performed with a simulated annealing method.

Acknowledgement We are grateful to the Natural Science Foundation of Zhejiang Province (No. Y2100908) for financial support.

\section{References}

1) Redinbo M. R., Stewart L., Kuhn P., Champoux J. J., Hol W. G. J., Science, 279, 1504-1513 (1998).

2) Koster D. A., Croquette V., Dekker C., Shuman S., Dekker N. H., Nature (London), 434, 671-674 (2005).

3) Rasheed Z. A., Rubin E. H., Oncogene, 22, 7296-7304 (2003).

4) Wall M. E., Wani M. C., Cook C. E., Palmer K. H., McPhail A. T., Sim G. A., J. Am. Chem. Soc., 88, 3888-3890 (1966)

5) Thomas C. J., Rahier N. J., Hecht S. M., Bioorg. Med. Chem., 12, 1585-1604 (2004).

6) Teicher B. A., Biochem. Pharmacol., 75, 1262-1271 (2008).

7) Hsiang Y. H., Hertzberg R., Hecht S., Liu L. F., J. Biol. Chem., 260, 14873-14878 (1985).

8) Staker B. L., Hjerrild K., Feese M. D., Behnke C. A., Burgin A. B. Jr., Stewart L., Proc. Natl. Acad. Sci. U.S.A., 99, 15387-15392
(2002).

9) Schaeppi U., Fleischman R. W., Cooney D. A., Cancer Chemother. Rep. 3, 5, 25-36 (1974).

10) Chrencik J. E., Staker B. L., Burgin A. B. Jr., Pourquier P., Pommier Y., Stewart L., Redinbo M. R., J. Mol. Biol., 339, 773-784 (2004).

11) Luzzio M. J., Besterman J. M., Emerson D. L., Evans M. G., Lackey K., Leitner P. L., McIntyre G., Morton B., Myers P. L., Peel M., Sisco J. M., Sternbach D. D., Tong W.-Q., Truesdale A., Uehling D. E., Vuong A., Yates J., J. Med. Chem., 38, 395-401 (1995).

12) Mi Z., Burke T. G., Biochemistry, 33, 10325-10336 (1994).

13) Meng L. H., Liao Z. Y., Pommier Y., Curr. Top. Med. Chem., 3, 305-320 (2003).

14) Morrell A., Antony S., Kohlhagen G., Pommier Y., Cushman M., J. Med. Chem., 49, 7740-7753 (2006).

15) Nagarajan M., Morrell A., Ioanoviciu A., Antony S., Kohlhagen G., Agama K., Hollingshead M., Pommier Y., Cushman M., J. Med. Chem., 49, 6283-6289 (2006).

16) Pereira E. R., Belin L., Sancelme M., Prudhomme M., Ollier M., Rapp M., Sevère D., Riou J. F., Fabbro D., Meyer T., J. Med. Chem., 39, 4471-4477 (1996).

17) Yamashita Y., Kawada S., Fujii N., Nakano H., Biochemistry, 30, 5838-5845 (1991)

18) Vicker N. Burgess L., Chuckowree I. S., Dodd R., Folkes A. J., Hardick D. J., Hancox T. C., Miller W., Milton J., Sohal S., Wang S., Wren S. P., Charlton P. A., Dangerfield W., Liddle C., Mistry P., Stewart A. J., Denny W. A., J. Med. Chem., 45, 721-739 (2002).

19) Thapa P., Karki R., Thapa U., Jahng Y., Jung M. J., Nam J. M., Na Y., Kwon Y., Lee E. S., Bioorg. Med. Chem., 18, 377-386 (2010).

20) Cho W. J., Min S. Y., Le T. N., Kim T. S., Bioorg. Med. Chem. Lett., 13, 4451-4454 (2003).

21) Pommier Y., Chem. Rev., 109, 2894-2902 (2009).

22) Mugnaini C., Pasquini S., Corelli F., Curr. Med. Chem., 16, 17461767 (2009).

23) You Q. D., Li Z. Y., Huang C. H., Yang Q., Wang X. J., Guo Q. L., Chen X. G., He X. G., Li T. K., Chern J. W., J. Med. Chem., 52, 5649-5661 (2009).

24) Al-Trawneh S. A., Zahra J. A., Kamal M. R., El-Abadelah M. M., Zani F., Incerti M., Cavazzoni A., Alfieri R. R., Petronini P. G., Vicini P., Bioorg. Med. Chem., 18, 5873-5884 (2010).

25) Ji Y. B., Zhou J. H., Zou M. X., You Q. D., Acta Pharmacol. Sin., 43, 811-818 (2008)

26) Kang D. H., Kim J. S., Jung M. J., Lee E. S., Jahng Y., Kwon Y., Na Y., Bioorg. Med. Chem. Lett., 18, 1520-1524 (2008).

27) Friesner R. A., Banks J. L., Murphy R. B., Halgren T. A., Klicic J. J., Mainz D. T., Repasky M. P., Knoll E. H., Shelley M., Perry J. K., Shaw D. E., Francis P., Shenkin P. S., J. Med. Chem., 47, 1739-1749 (2004).

28) Staker B. L., Feese M. D., Cushman M., Pommier Y., Zembower D., Stewart L., Burgin A. B., J. Med. Chem., 48, 2336-2345 (2005).

29) Fox B. M., Xiao X., Antony S., Kohlhagen G., Pommier Y., Staker B. L., Stewart L., Cushman M., J. Med. Chem., 46, 3275-3282 (2003). 